\title{
Information Dashboards
}

- Dashboard Design

- Team Science Approach

- $\quad$ Project Milestones \&

- WBS \& Budgets

- Working Dashboards

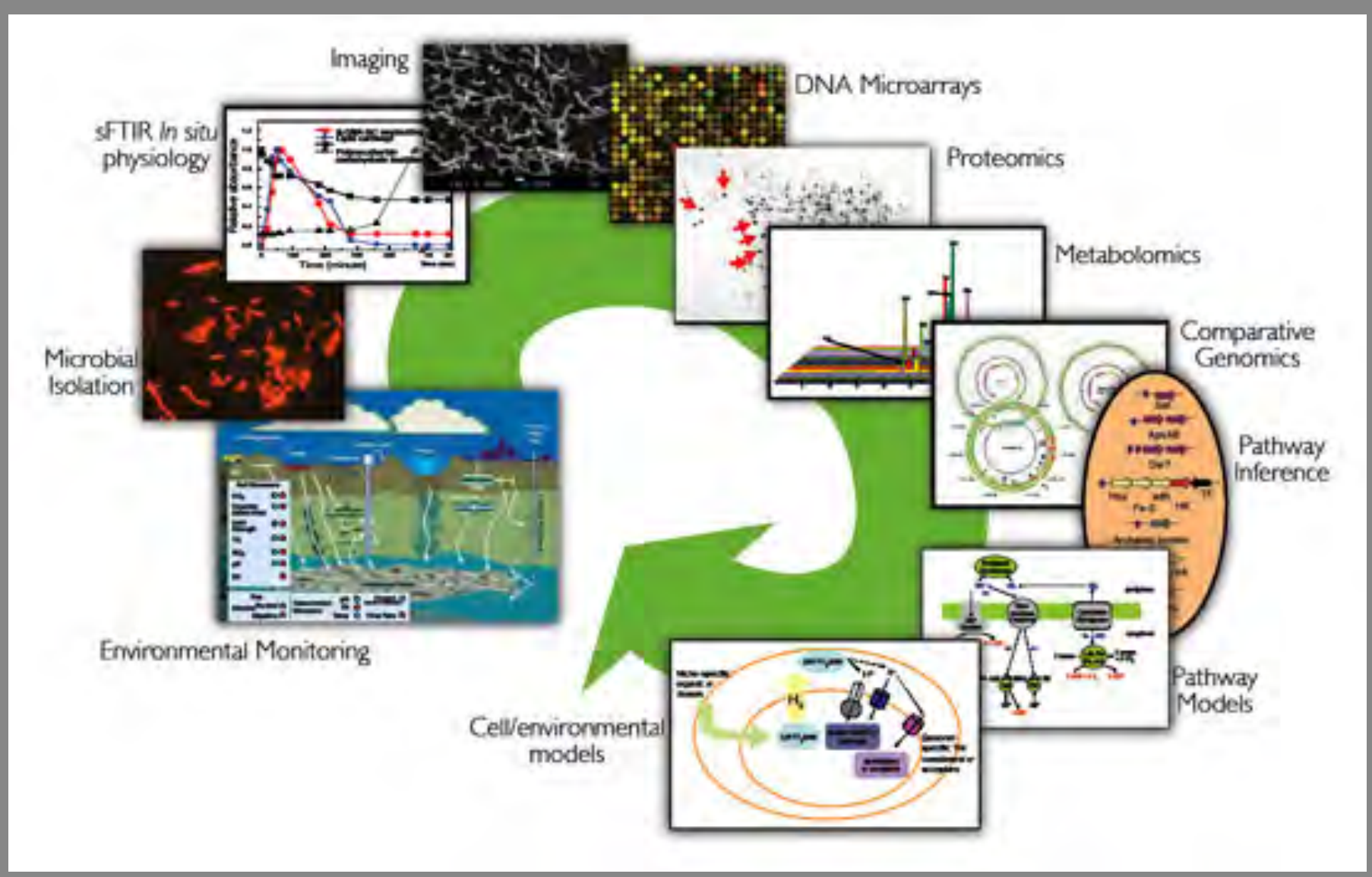

Amy Shutkin, CPM

ashutkin@lbl.gov

Ernest Orlando Lawrence Berkeley National Laboratory

Physical Biosciences Division

ACKNOWLEDGEMENT

ESPP2 is part of the Virtual Institute for Microbial Stress and Survival supported by the U. S. Department of Energy, Office of Science, Office of Biological and Environmental Research, Genomics Program:GTL through contract DE-AC02-05CH11231 between Lawrence Berkeley National Laboratory and the U. S. Department of Energy. 
INFORMATION DASHBOARD DESIGN

The Effective Visual Communication of Data
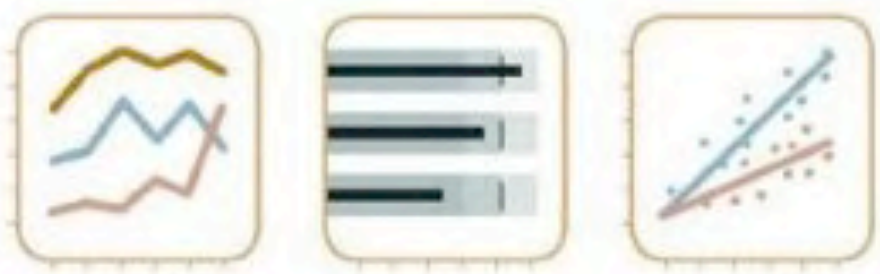

Stephen Few

O'REILLY*

\section{About the Author}

Stephen Few has worked for over 20 years as an IT innovator, consultant, and teacher. Today, as Principal of the consultancy Perceptual Edge, Stephen focuses on data visualization for analyzing and communicating quantitative business information. He teaches in the MBA program at the University of California, Berkeley. You can learn more about Stephen's work and access an entire library of articles at

www.perceptualedge.com. 


\section{Dashboards - defined}

"A dashboard is a visual interface that provides at-a -glance views into key measures relevant to a particular objective or business process."

Key Attributes:

$\checkmark$ Graphical to focus attention on key trends, comparisons and exceptions

$\checkmark$ Display only relevant data

$\checkmark$ Inherently contain predefined conclusions

Note: Collecting user requirements is KEY
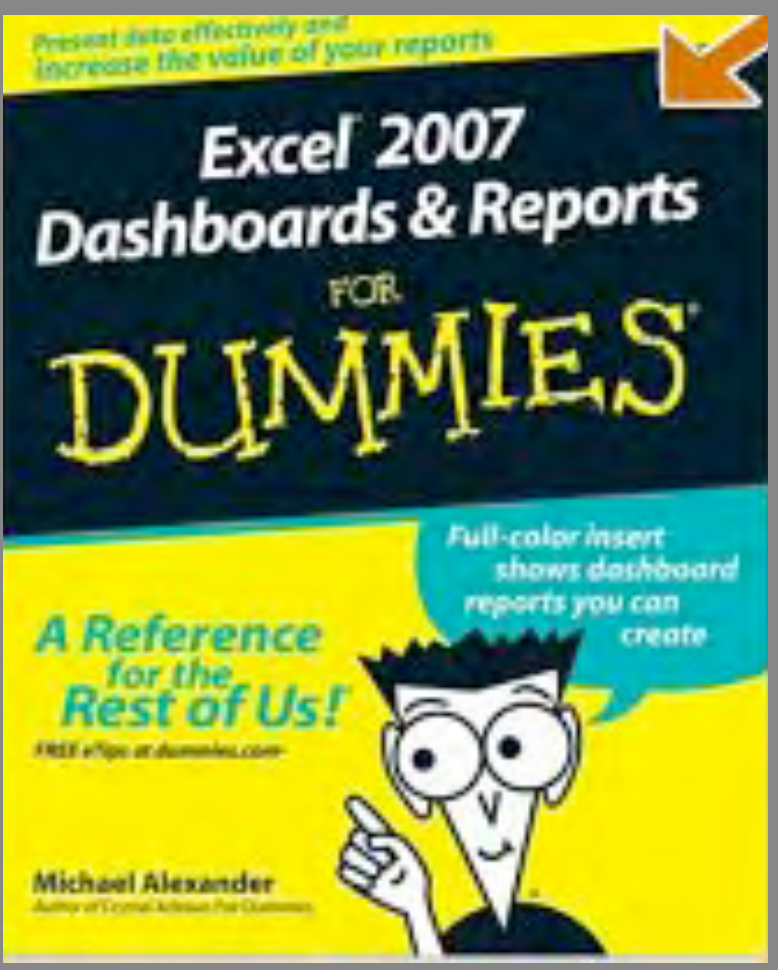

from 'Excel 2007 Dashboards \& Reports for Dummies' by Michael Alexander 


\section{Dashboards - bad examples}

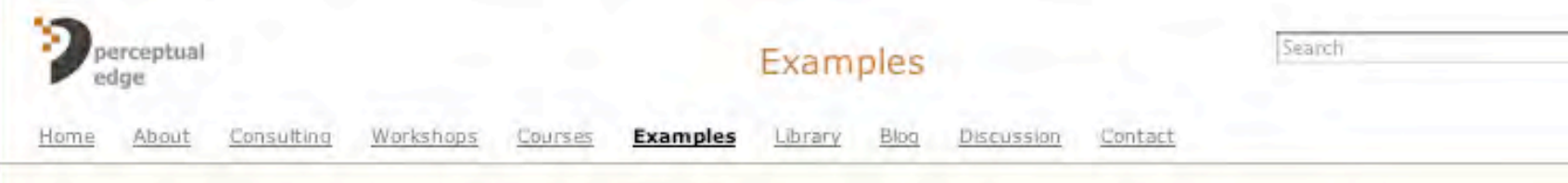

Each of the examples that ap pear below illustrates quantitative information that is poorly designed for communication. Click on any of these examples to see an analysis of its problems and my proposed solutions.
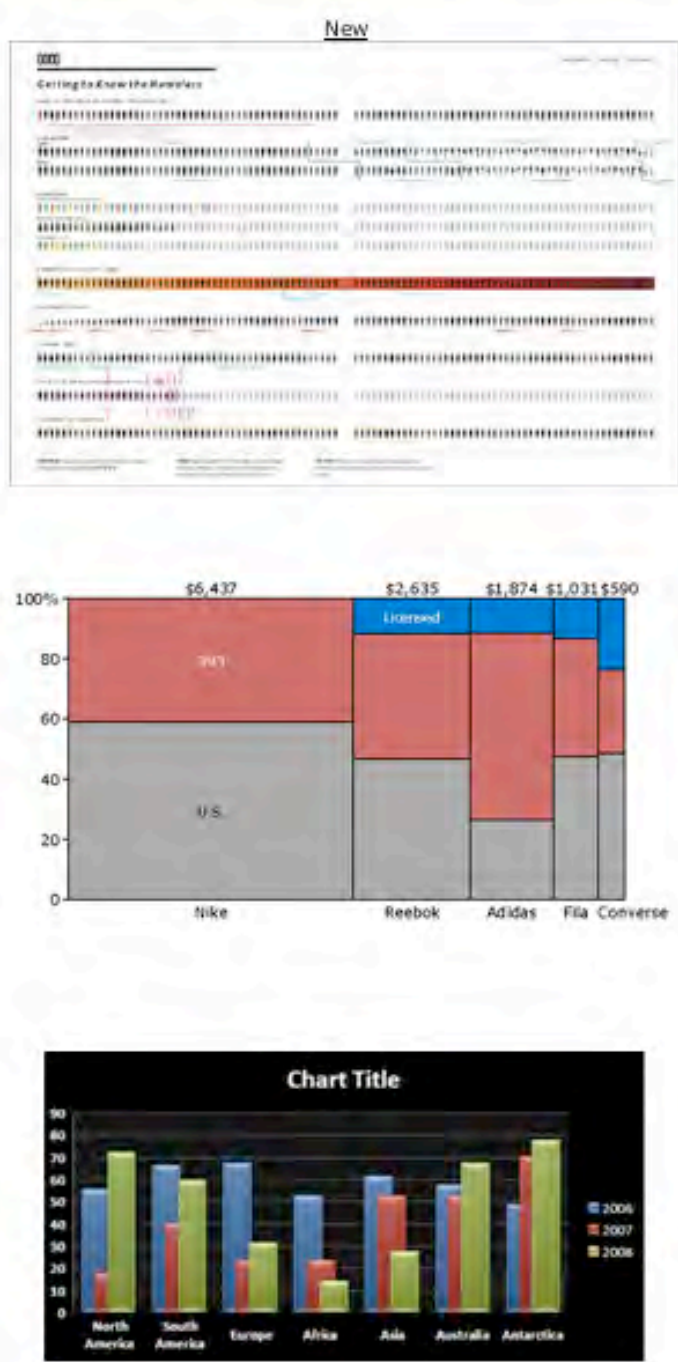

New

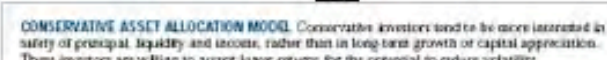

6x iternersal

28 incap

axingecip

is constrosin tquasere

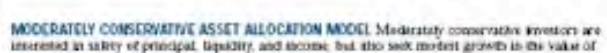

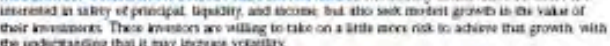

125 inerunoral

32 isicape

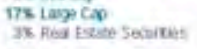

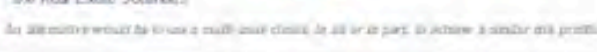
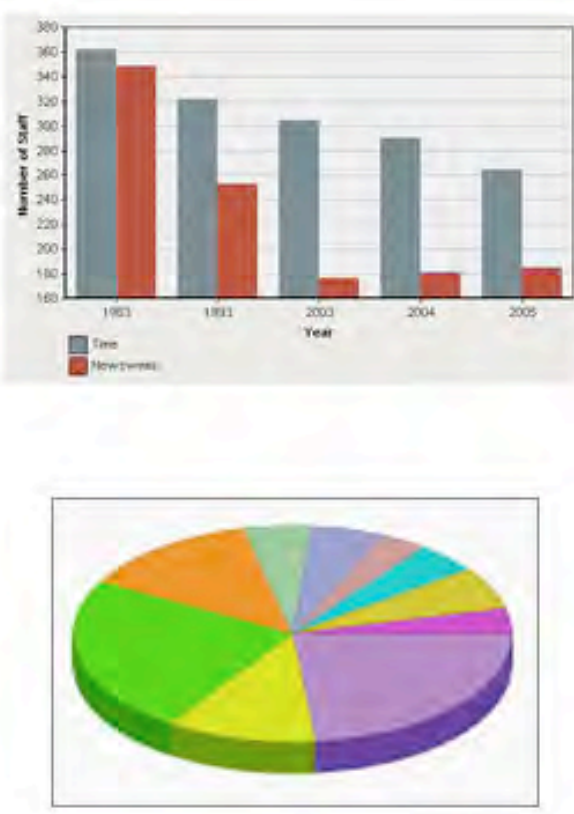

Common Problems:

Positioning content in places that don't fit its importance

Positioning content in places that fail to support its use

Including items that serve no useful purpose

Sizing content larger than it deserves

Separating content excessively

Visually featuring content \& other items more than they deserve

Failing to link contents \& other items that are related

Visually suggesting links between unrelated content Enforcing a rigid symmetrical grid 


\section{Dashboards - bad examples}

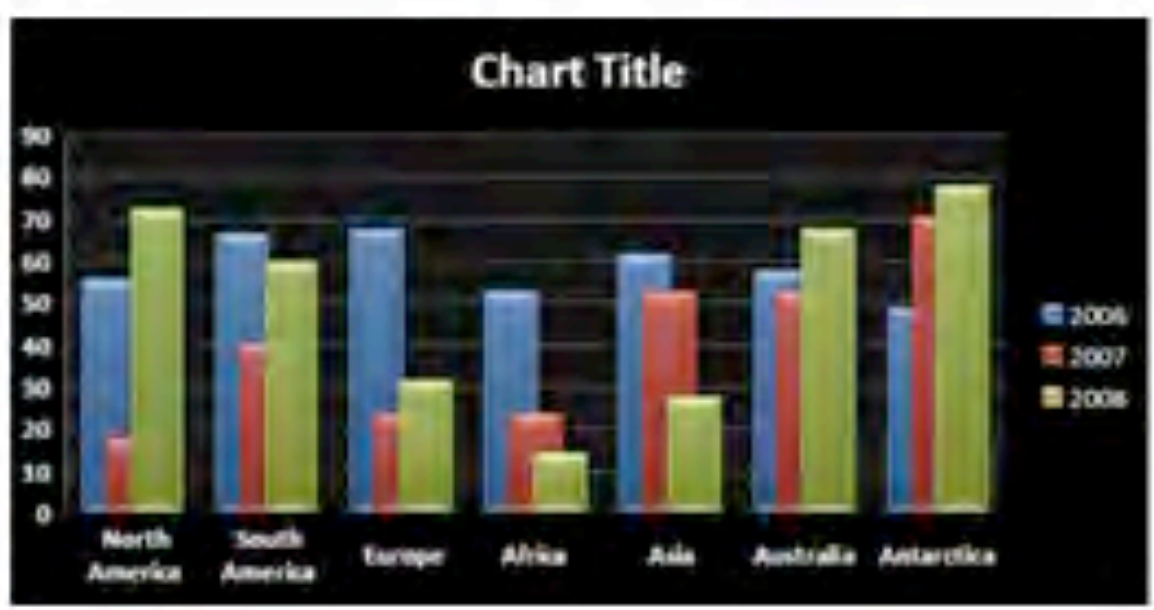

Here are a few of this graph's problems:

- There are several distracting (and detracting) visual effects: the reflection of light, transparency, and 3-D effects on the bars (and squares in the legend) add no value.

- The bars have been overlayed on one another, which partially obscures the first two sets and gives them different visual salience. Because the bars for the year 2008 appear in the forefront of each cluster, their greater importance is implied, which was probably not intended. While I can't be sure, the graph's original post date of 2005, suggests that these values are projections, albeit unbelievably volatile ones. Without knowing more about the data, I can't say for sure, but the 2006 projections are probably the surest and most relevant, yet they are partly obscured by the other two years.

- Although the gridlines in this graph are thin and light, because these values are projections, we probably don't need to know precise values. As such, the gridlines are not necessary.

- The bar colors are more intense than they should be. The use of high-intensity colors should be reserved for making important data salient. Regular data should be shown using less intense colors. After all, when you display all of your data to stand out, nothing does.

- The continents have not been ordered in a logical way. At the very least they could have been alphabetized, but, as we'll see below, there's almost always a better way to order your data.

- Although bar graphs are great for showing and comparing the magnitudes of different variables, they are inferior to lines for showing how the values change through time. Because the pattern of change through time is likely more important than the actual magnitudes of the individual values, a line graph would have worked better. 


\section{ESPP 2 \\ Environmental Stress}
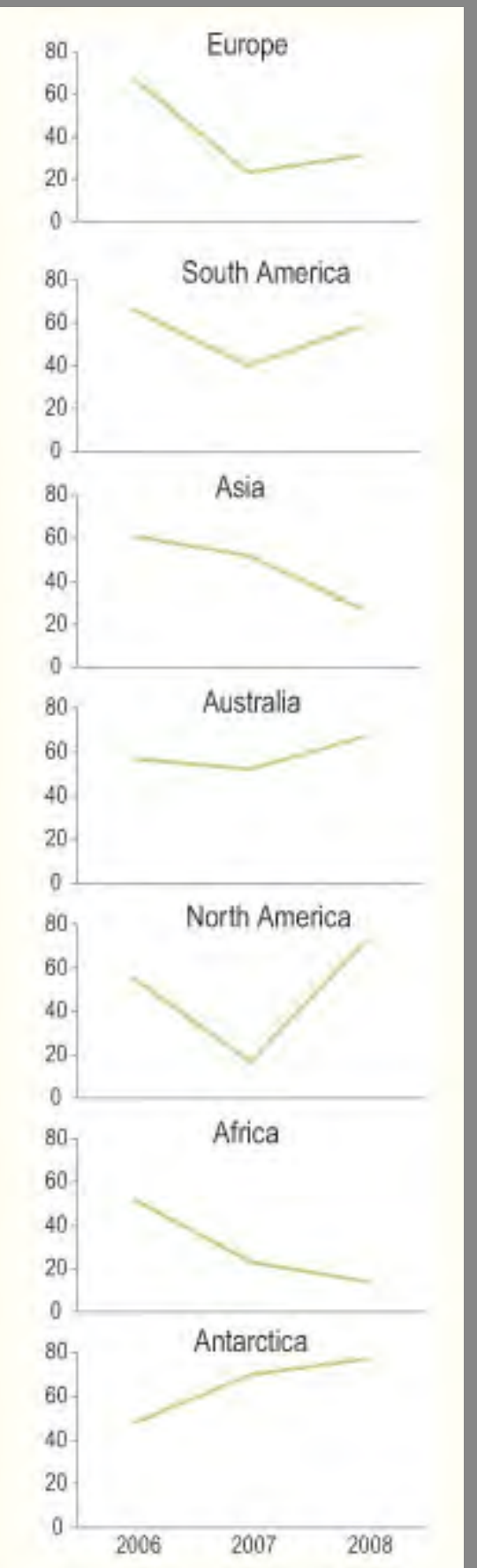

\section{Dashboards - solutions}

Line graphs make it especially easy to see the patterns of change and to focus on trends. To avoid the clutter of seven lines on a single graph, I used "small multiples," a series of seven small graphs, which vary by region, but otherwise look and work the same. Small multiples may be arranged vertically (shown above), horizontally, or in a matrix. Because this information is a projection (and so the exact magnitudes are probably not as important), I have made the assumption that the graphs should be arranged to make it easiest to compare the patterns of change for the various regions, which is why I aligned the years by arranging the graphs vertically. If the magnitudes of the lines were more important, then a horizontal layout would have been preferable, for easier magnitude comparisons. Notice that the horizontal label (showing the years) is only shown on the very bottom of the graph. This is all that's necessary to show which part of each line belongs to which year. Duplicating these labels for each graph would have resulted in redundancy and clutter.

I have reordered the continents based on the 2006 values, with the highest at the top and the lowest at the bottom. I based the sequence on the 2006 value because, as these values are projections, the first year is likely to be most reliable and of greatest interest to decision-makers.

This new design is clean and clear-free of the visual distractions in the first two examples. Anyone viewing the graph would be able to examine the data, focusing perhaps on the large declines that are projected to occur in Europe and Africa, instead of the pretty, shiny bars.

Reduce the non-data ink Enhance the data ink 
Let's examine another ineffective use of pie charts. Edward Tufte once said that "the only worse design than a pie chart is several of them, for then the viewer is asked to compare quantities located in spatial disarray both within and between pies ${ }^{n}$ (Edward Tufte, The Visual Display of Quantitative Information, Graphics Press, 1983, p. 178.) I share Tufte's opinion that this is an ineffective way to compare multiple part-to-whole relationships.
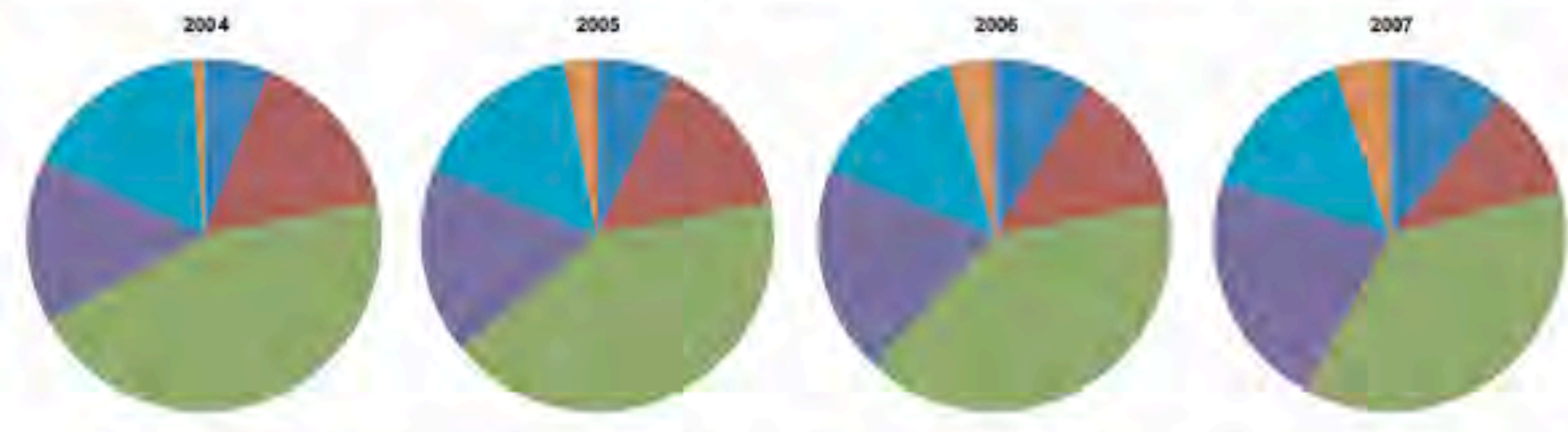

mompry $\mathrm{A}$

mCompary $\mathrm{H}$

icompry C

wCompory $\mathrm{D}$

mCompry E

acompary F

Try to follow the changes of these various companies and how they compare to one another through time. It is nearly impossible. Notice how easily you can do it, however, using the following display:
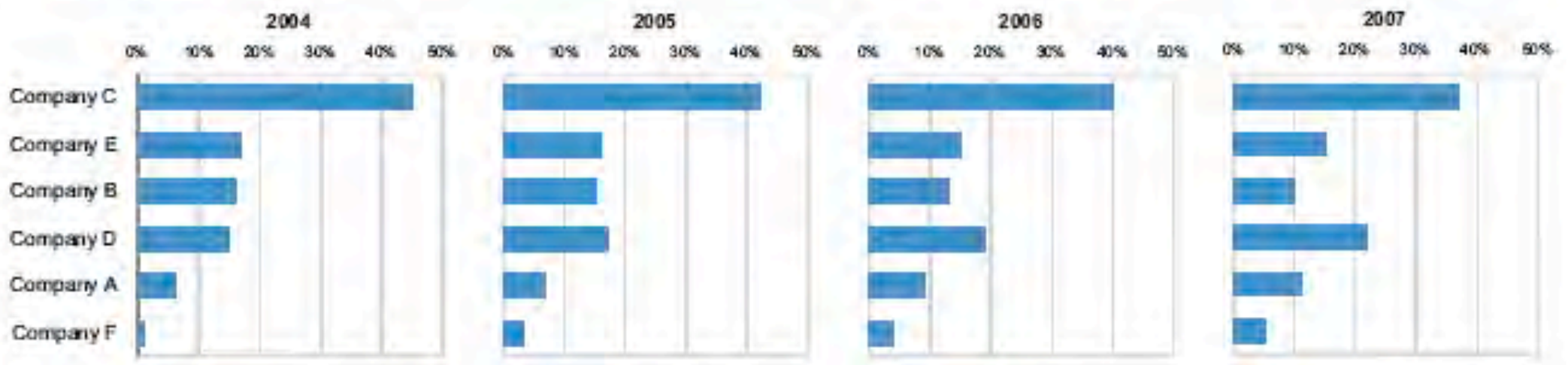
The data is great but the display is a jumbiad mess.

$\wedge$ solution

Here's the same data displayed simply and clearly:

\section{Percentage of Analytic Computer Usage by Type}

\section{Percent of Enterprise} 4.25\% EProducer

Extended Enterprise User (Extranets/E2B/B2C and mabilei wireless)

Casual User

(Dashboards and enterpicse reporting)

Business User

(Scorecards, performance mgnt, business reporting, and packaged apps)

Power User
Statistical analysis, analylical reporting

and OLAP

iT User

(Appligatinn development, data mining and meta data desigh)
Reduce the non-data ink

Enhance the data ink

I could have uged colors but, frankik, this graph doesnt need themr Limiturg it to black and white allows vou to

photacopy this usefur infarmation and pass, it an without any lass af quality, Can you imagine whiar the original pie chiart would look like if you photocopied if in black and white? 


\section{Dashboards - real estate}

"The relative prominence of screen space on a dashboard can be divided into quadrants... Whenever possible, place information that is considered most important in the upper left hand region and that which is least important in the lower right hand corner."

\section{Emphasized}

\section{Neither emphasized nor de-emphasized}

Neither emphasized

Emphasized nor de-emphasized

\section{De-emphasized}




\section{Team Science Approach}

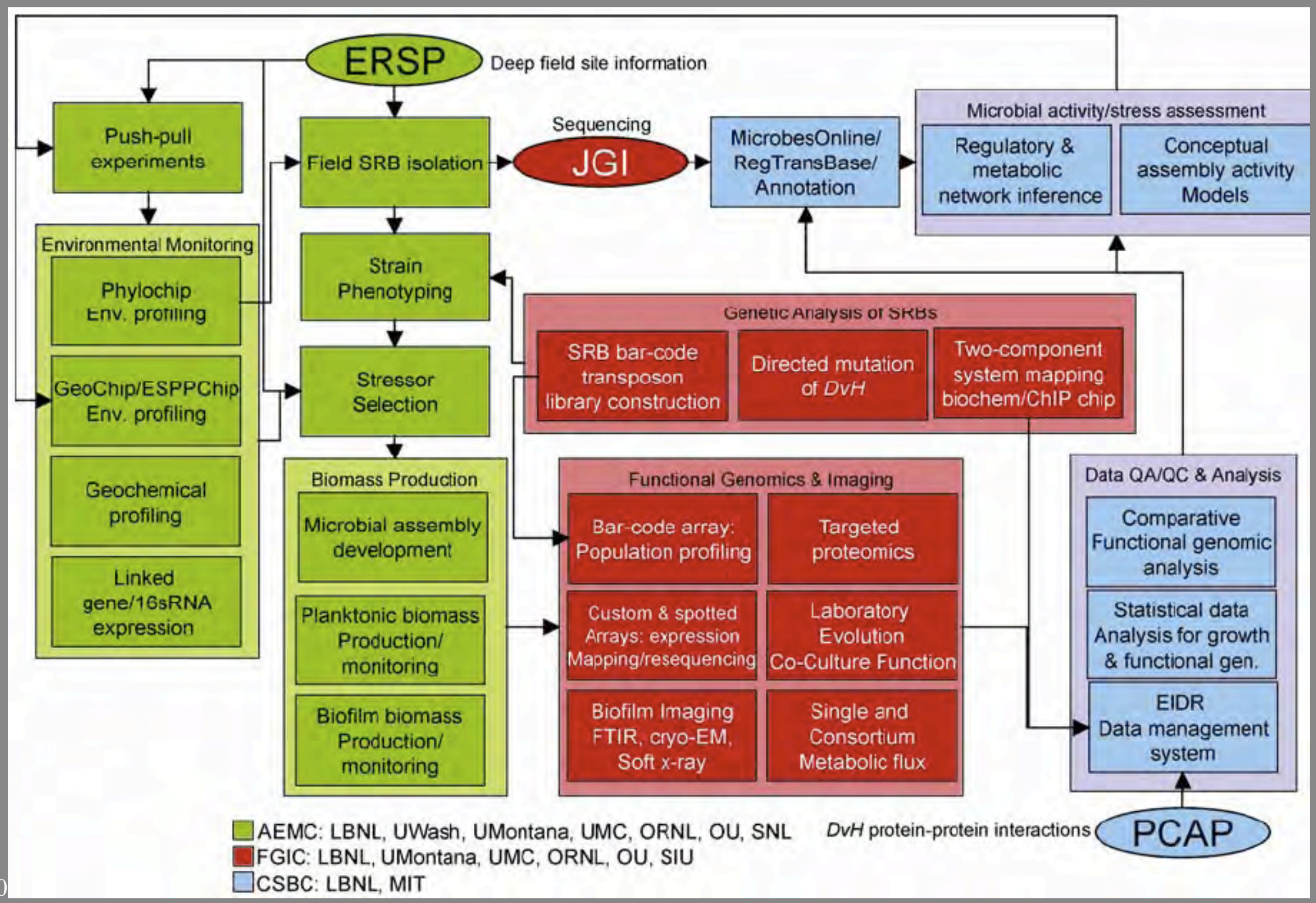




\section{Team Science Approach}

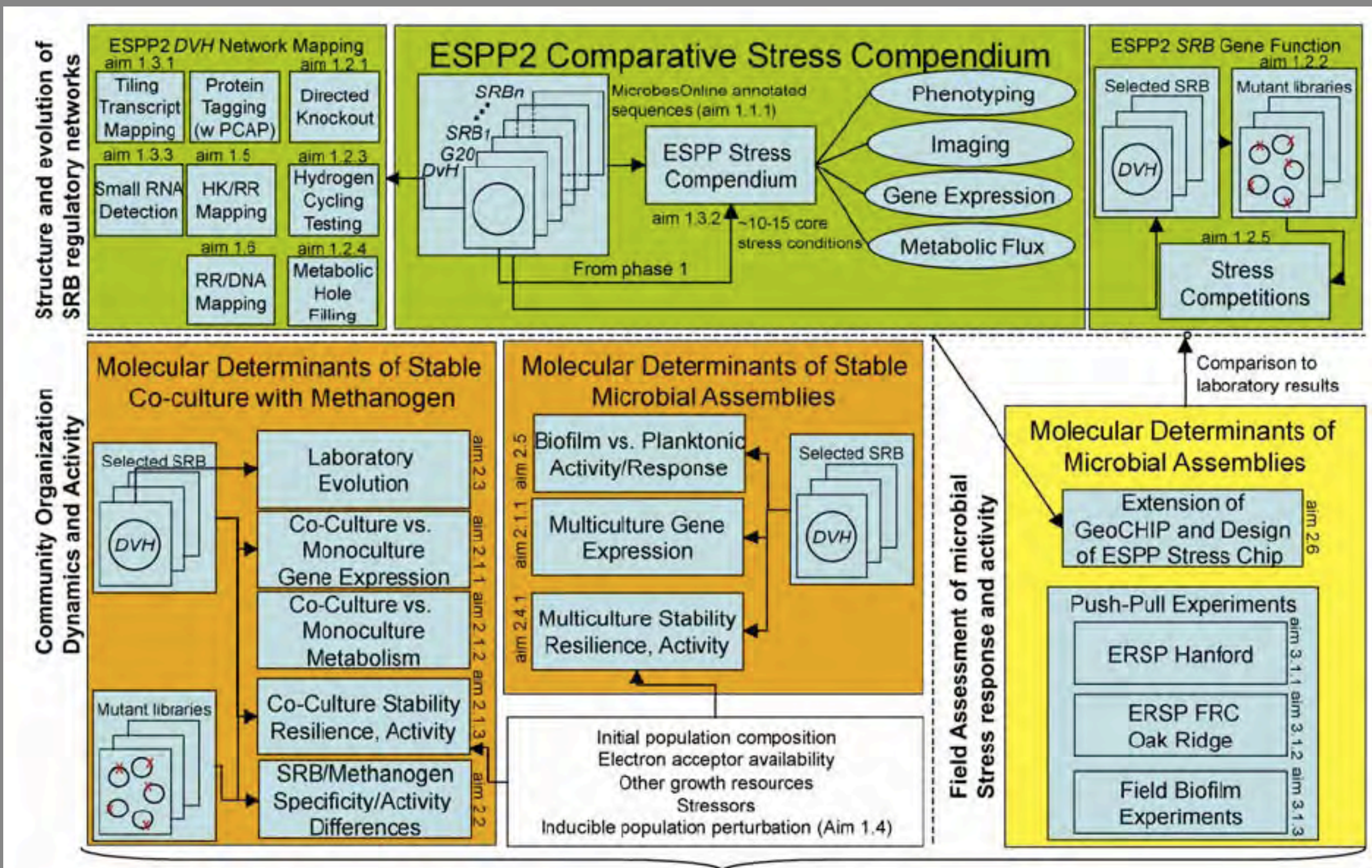




\section{Milestones: Year One 09/30/2008}

AEMC

-Obtain previously isolated SRB (especially for DOE contaminated sites), prepare DNA for sequencing submit to JGI. - Growth optimization and stability studies of different syntrophic co-culture assemblies: Alternative Dv strains/species. -Full scale biomass production for steady-state growth stress-perturbed co-culture response experiments (perturbation \& steady state analyses using optimized co-culture conditions) for different SRB/methanogen pairs.

-Initial tests of multiculture conditions.

-Initiation of co-culture evolution experiments.

-Optimize transposon strain library competition experiments for read-out by bar code arrays both in monoculture and co -culture.

-Complete membrane profiling of $D$. vulgaris and M. maripaludis in mono culture and together in syntrophic culture. -Design of push-pull experiments and initial characterization of site bacterial populations and geochemistry and Hanford and Oak Ridge, including initial testing of in well sediment/attachment simulation systems.

-Design larger scale attached stress experiments for comparison with planktonic experiments (transcriptomics).

-Complete contrast/compare studies of groundwater and sediment ecogenomics from Oak Ridge site for metagenome (Sanger, 454, and clone libraries), 16SRNA Phylochip, Geochip, and realtime Q-PCR.

$\underline{\text { FGIC }}$

-Create and sequence-verify saturating tagged transposon library of $D$. vulgaris and $D$. alaskensis G20.

-Prioritize HK/RR pair characterization with Computational Core.

- Tag and purify HK/RR pairs.

-Initial HK/RR mapping by biochemical assay.

-Initial proof of concept RR/DNA mapping using ChIP-chip.

- Optimize barcode array design.

-Optimize tiling array for transcription start-stop mapping, small RNA detection and ChIP-chip in SRB for G20 and DvH.

-Optimize multiplex gene expression design for G20.

-Complete stress response transcriptomics for G20.

-Initial survey of possible small RNA regulators.

-Complete design and testing of ESPPChip microarray.

$\underline{\text { CSBC }}$

•Extension of MicrobesOnline for 16SRNA, GeoCHIP/ESPPChip, Phenotype, metagenomic data.

-Complete computational analysis of DvH and G20 and methanogen metabolism.

-Establish flux model analysis methods for mono- and multicultures.

-Developing tiling array and bar-code array design and analysis techniques.

-Complete annotation of Dv Miyazaki, Ds 27774, and one Dv Hanford isolate.

-Complete initial reannotation of DvH.

•Begin design of conceptual model of stress, ED, TEA responses for Hanford Cr and Oak Ridge U contaminated sites. 
ESPP2 Work Breakdown Structure by Milestones

Schedule Development \& Execution

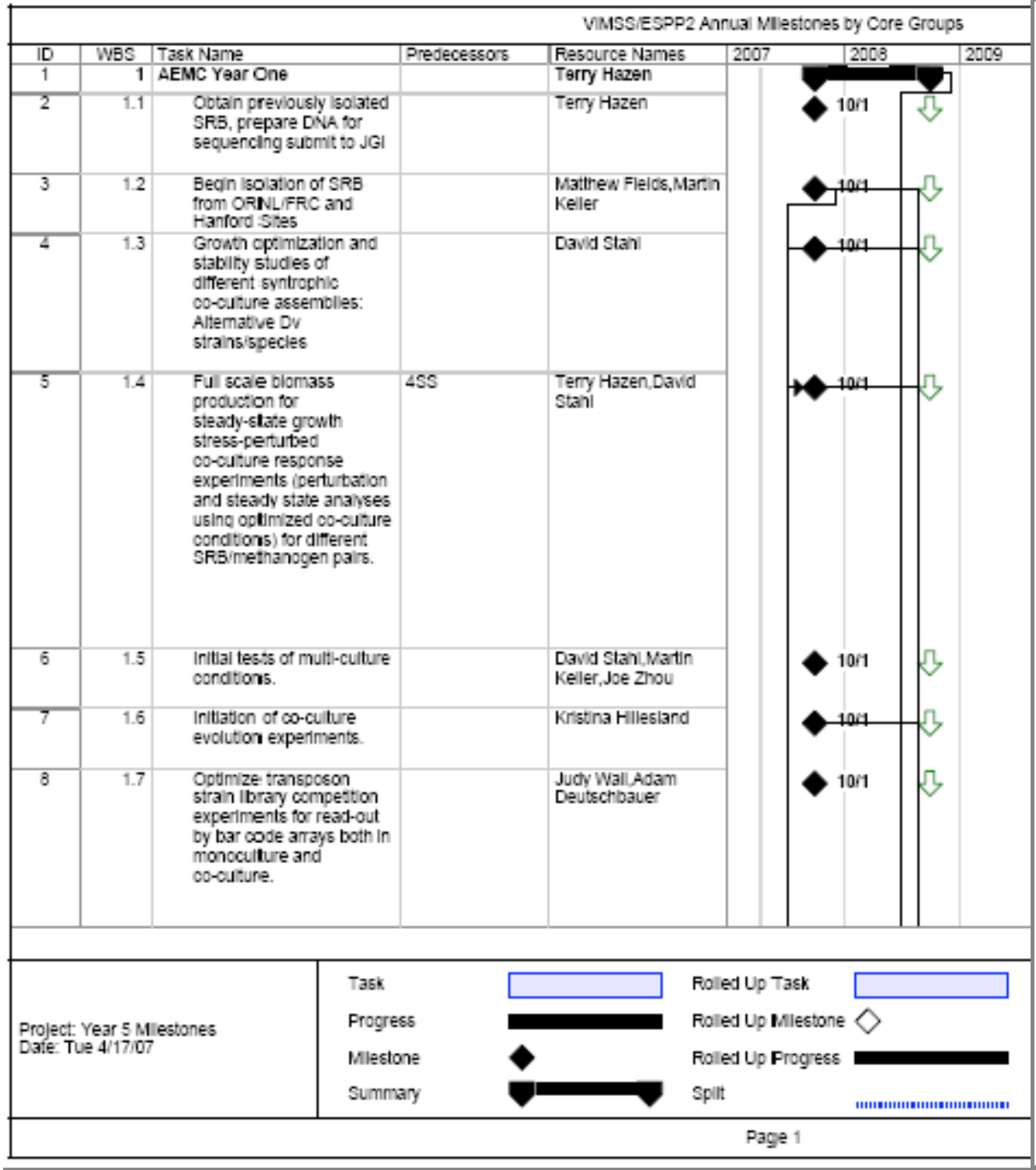

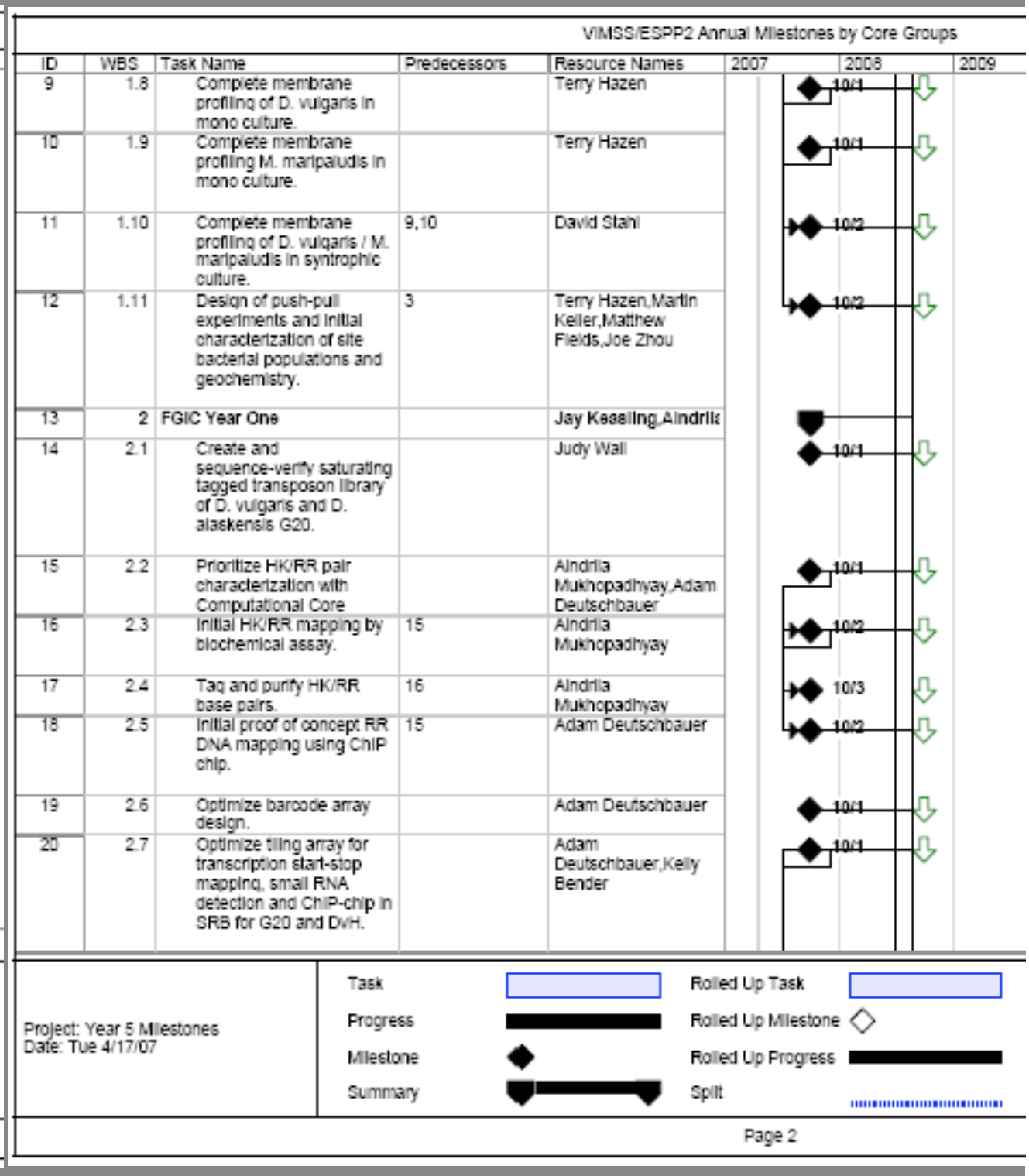




\section{Dashboard Milestone Reports}

\section{FY08 Milestones: AEMC}

Obtain previously isolated SRB, prepare DNA for JGI sequencing.

Syntrophic co-culture assemblies: Growth optimization \& stability studies: Alternative Dv strains/species. BMP: steady-state growth stress-perturbed co-culture response experiments for different SRB/methanogen pairs.

Initial tests of multiculture conditions.

Initiation of co-culture evolution experiments.

Optimize monoculture and co-culture transposon strain library competition experiments for bar code array read-out.

Complete membrane profiling of $\mathrm{D}$. vulgaris and $\mathrm{M}$.

maripaludis in mono culture and in syntrophic culture. Design push-pull experiments \& initial characterization of site bacterial populations and geochemistry @ Hanford \& ORNL, including initial testing of in well sediment/attachment simulation systems.

Design larger scale attached stress experiments for comparison w/ planktonic *experiments

(transcriptomics).

Complete contrast/compare studies of groundwater and sediment ecogenomics from Oak Ridge site for

metagenome (Sanger, 454, and clone libraries),

16SRNA Phylochip, Geochip, and realtime Q-PCR.

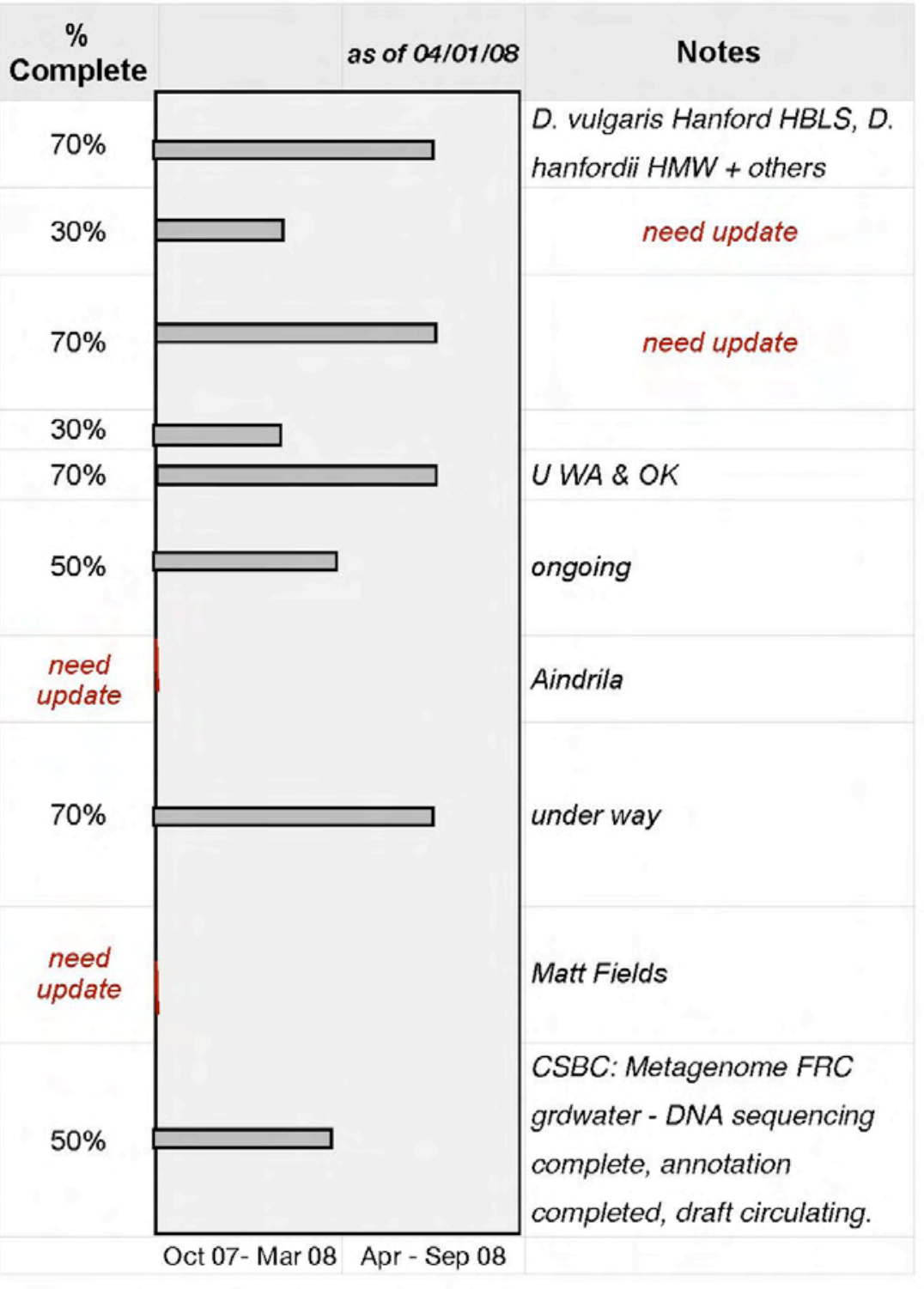

\section{Key Performance Indicators (KPI)}




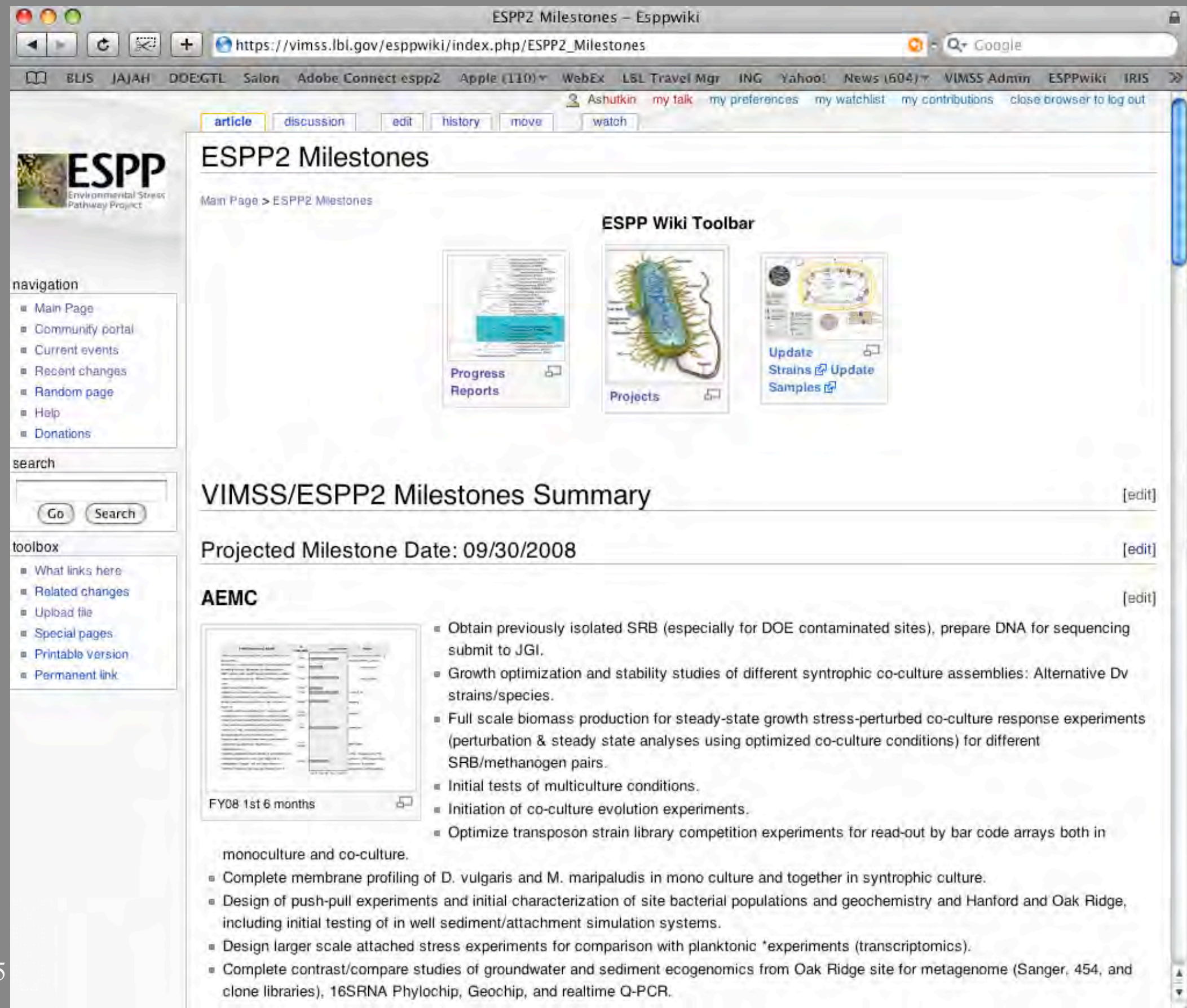




\section{Dashboard Milestone Reports}

\section{FY08 Milestones: AEMC}

Obtain previously isolated SRB, prepare DNA for JGI sequencing.

Syntrophic co-culture assemblies: Grovah oplimization

\& stability studies: Alternative Dv strainsispecies.

BMP: steady-state growth stress-perturbed co-culture

response experiments for different SRBimethanogen

pairs

Initial tests or multiculture conditions.

Initiation of co-culture evolution experiments.

Optimize monocuiture and co-culture transposon strain

library competition experiments for bar code array

read-out.

Complete membrane profiling of $\mathrm{D}$. vulgaris and $\mathrm{M}$.

maripaludis in mono culture and in syntrophic culture.

Design push-pull experiments \& Inittal characterization

of site bacterial populations and geochemistry (c)

Hanford \& ORNL., including initial testing of in well

sediment/attachment simulation systems

Design larger scale attached stress experiments for

comparison w/ planktonic "experiments

(transcriptomics).

Complete contrasticompare studies of groundwater and

sediment ecogenomics from Oak Ridge site for

metagenome (Sanger, 454, and clone libraries)

16SRNA Phylochip, Geochip, and realtime Q.PCR.

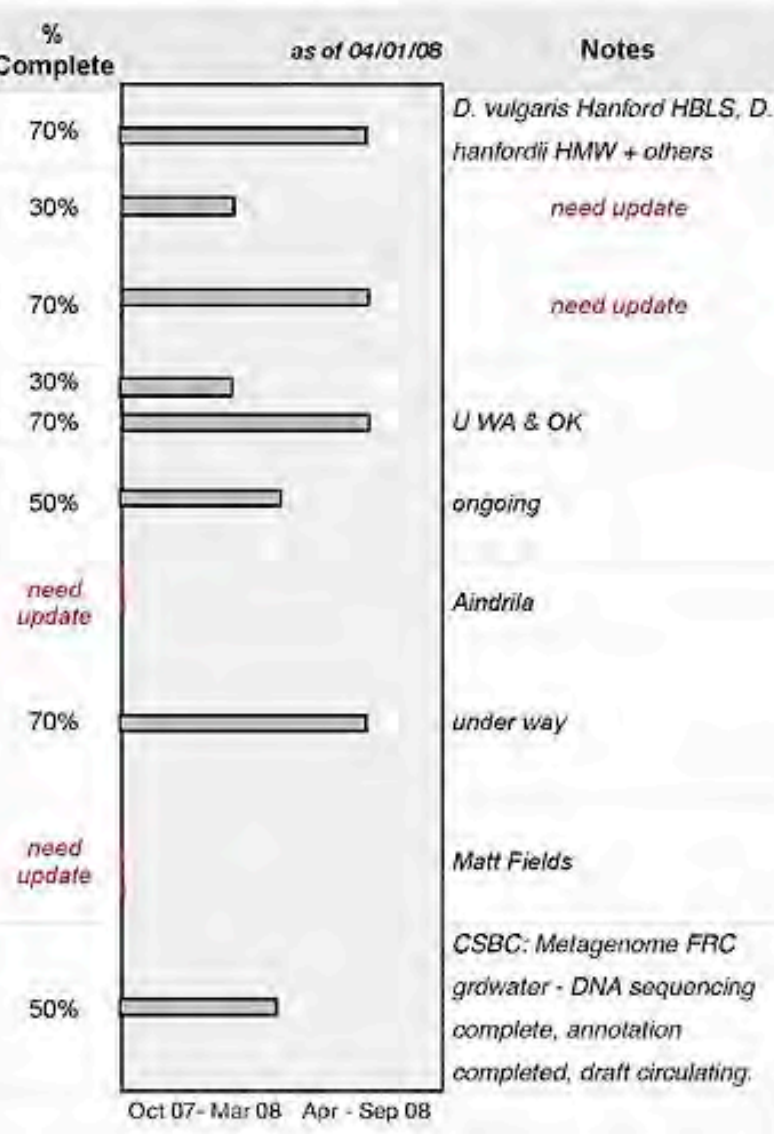

$\%$ 
Rate Type

Escalation Rates

LBNL Labor

LBNL Supplies \& Other

Expenses (OMB)

Total Labor

Original Project Budget

Assumptions

Total Supplies \&

Other Expenses

(OMB)

Totals

LBNL Labor

Equipment, Supplies \& Other Expenses, LBNL

(OMB)

LBNL Tota

Renewal

Budget

Assumptions

Total Direct Costs, LBNL

Total Indirect

Costs, LBNL

Total Direct Costs less Other Inst.

Indirect Costs

LBNL

SNL

ORNL

ESPP2 Total

17

$\%$ original projection
$\underline{F Y 07} \quad \underline{F Y 08} \quad \underline{F Y 09} \quad \underline{F Y 10}$

\section{$\underline{\text { FY11 }} \underline{\text { FY12 (est) } 5 \text { Year Totals }}$}

LBNL Forward Pricing Rates

Effective October 1, 2006

Revision 5

Rate Type

Escalation Rates

Labor

Supplies \& Other Expenses (OMB)

Construction Projects (OECM)

Institutional Rates

General and Administrative

G\&A (Off Site) Rate - OFF

Site Support (Fabrication) Rate - FAB

Gretina

Animal Care

General Rate - GR1

LDRD Rate

LDRD Operating and Equipment

(LDRD rate is based on proposed structure, which is currently being reviewed by DOE)

IGPP Rate

IGPP

Procurement Burdens (Base: Cost of procured materials \& services) PO's $\$ 1-\$ 500,000$

PO's Over $\$ 500,000$ (per PO)

$R \& D$ subcontract's $\$ 1-\$ 300,000$

R\&D subcontract's over $\$ 300,000$

Intra-University Transfers (IUTs) $\$ 1-\$ 200,000$

Intra-University Transfers (IUTs) over $\$ 200,000$

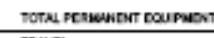

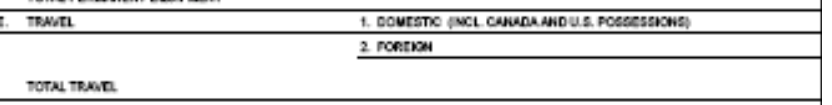

Genomics Procurements (PO's $\$ 1-\$ 500,000)$

Molecular Foundry (PO's $\$ 1-\$ 500,000)$

Project Management (PO's \$1-\$500,000)

Travel (Base: Travel Costs)

$$
\text { Travel Rate }
$$

Payroll Burden (Base: Delivered effort cost only)

Career\&Term Employees

Post Docs, Visiting Post Docs, Limited Employees, and Visting Researchers GSRAs

Students/Rehired Retirees/Employees working variable time

Summer Faculty

Fringe Benefits Only (Base: FTE gross pay only)

Post Docs, Visiting Post Docs, Limited Employees, and Visting Researchers GSRAs

Students/Rehired Retirees/Employees working variable time

Please sec the indinect calculation sheets

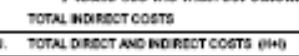

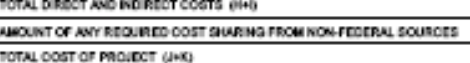

Summer Faculty 


\section{Dashboard} Integrated Milestone \&

\section{Budget Reports}

"How then do we make it easy for people to compare related sets of values when they are associated with different units of measure? Two answers come to mind. The first and most obvious is to place them in separate graphs, positioned close to one another so that the patterns in each can be compared to one another, but magnitude comparisons will be discouraged." Stephen Few light=budget available dark=current burnrate ytd budget variance

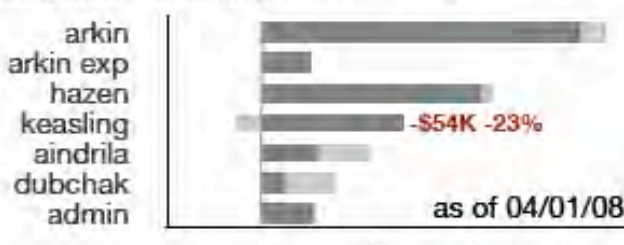

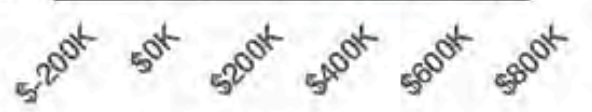

$\$ 1,500 \mathrm{~K}$

$\$ 1,250 \mathrm{~K}$

$\$ 1,000 \mathrm{~K}$

$\$ 750 \mathrm{~K}$

$\$ 500 \mathrm{~K}$

$\$ 250 \mathrm{~K}$

$\$ O K$

$\$ 1,500 \mathrm{~K}$

$\$ 1,200 \mathrm{~K}$

$\$ 900 \mathrm{~K}$

$\$ 600 \mathrm{~K}$

$\$ 300 k$

sok

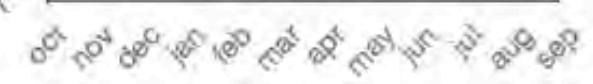

PI wiki Critical Events, Milestones \&

update Top Projects

Arkin - intemal $3 / 25 / 08$ Metabolic profile analysis

for others $3 / 25 / 08$ Experimental planning

Hazen

Keasling 3/31/08 Co-culture Biomass, IMS

Mukhopadhya

3/12/08 Flux work

Alm

Dubchak

Fieids

Keller

Singh

Stahl

Wall

Bender

Zhou

Sequencing
3/24/08 HK knock-outs, HK/RR

2/14/08 Compendium analysis, FRC

3/25/08 Integrate, annot. metab. pthway

2/18/08 DvH Cr transcriptomics: biofilm

3/10/08 Env mRNA profiling, consortia

3/7/08 Tri-culture \& Mesocosm

2/29/08 Methanococcus transcriptional

proteomic/mutant analysis in

proteormic

$1 / 31 / 08$ Deletions via marker xchange

2/7/08 Small RNA's - ongoing

$3 / 6 / 08$ Evolution, Hanford, FRC

9/7/07 Need update
$\$ 580.20 \mathrm{~K}$

$\$ 464.16 \mathrm{~K}$

$\$ 348,12 \mathrm{~K}$

$\$ 232.08 \mathrm{~K}$

$\$ 116.04 \mathrm{~K}$

$\$ O K$

$\$ 550 \mathrm{~K}$

$\$ 440 K$

$\$ 330 \mathrm{~K}$

$\$ 220 \mathrm{~K}$

$\$ 110 \mathrm{~K}$

\$OK

$\$ 300 \mathrm{~K}$

$5225 \mathrm{~K}$

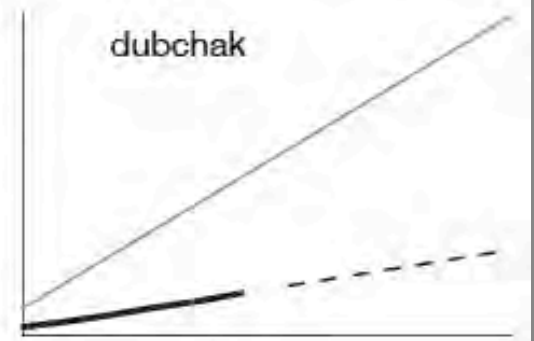

\$OK

$\$ 300 K$

$\$ 225 \mathrm{~K}$

$5150 \mathrm{~K}$

$\$ 75 K$

sok

$\$ 200 k$

$\$ 150 \mathrm{~K}$

$\$ 100 \mathrm{~K}$

$\$ 50 \mathrm{~K}$

sok

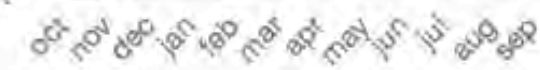

\section{admin}
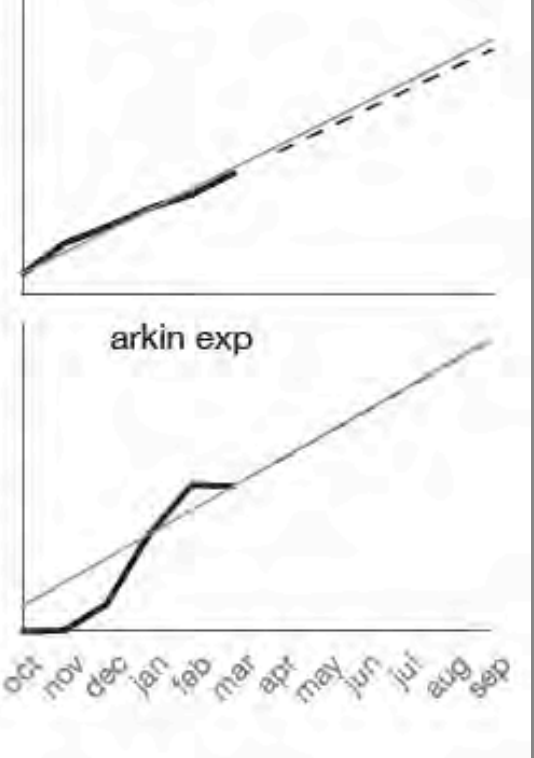


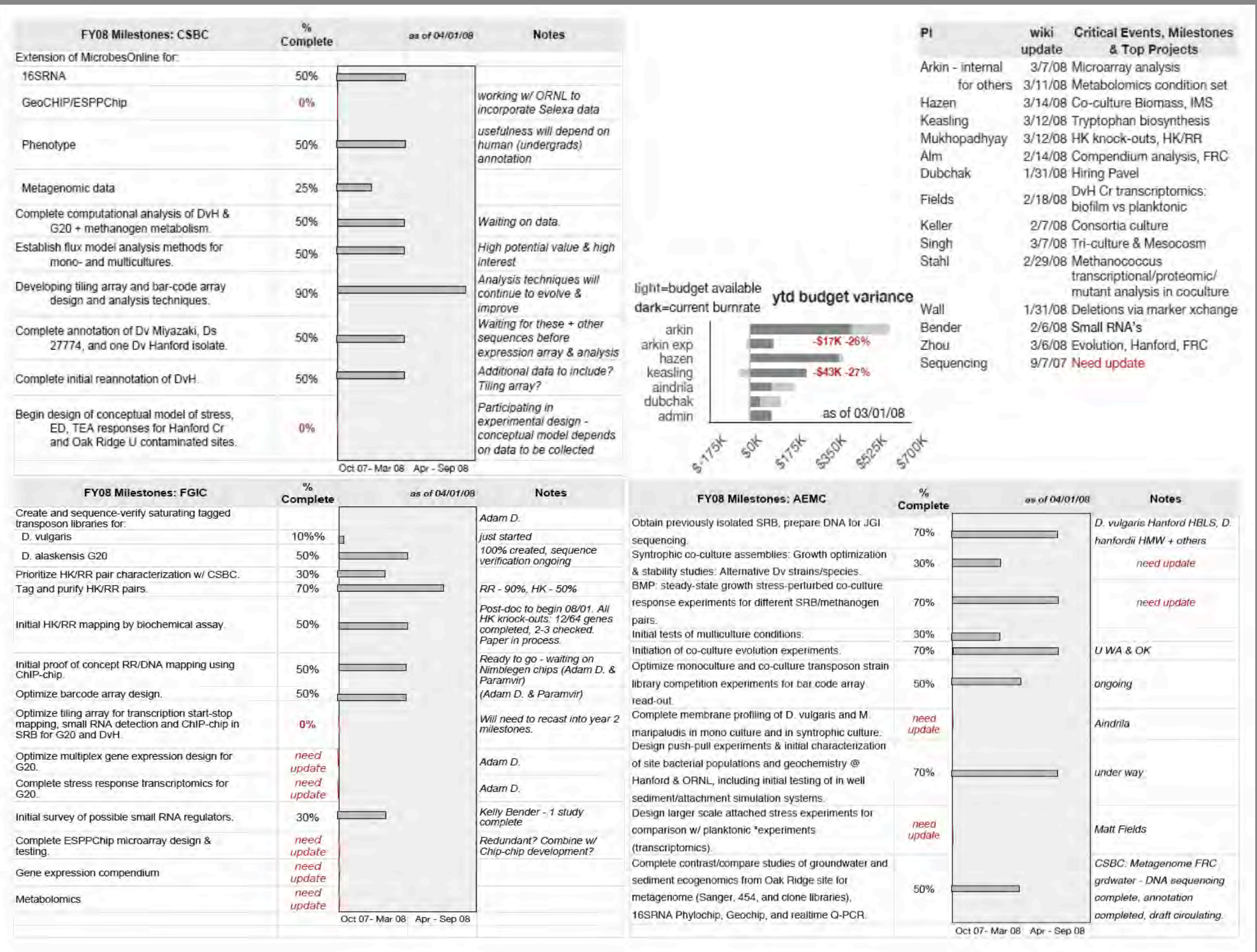




\section{Acknowledgements}

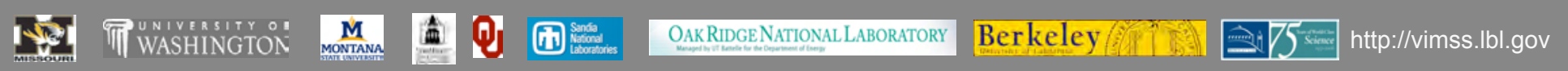

\section{Adam P. Arkin and Terry C. Hazen, Directors}

Applied Environmental Microbiology Core:

- LBNL, Terry C. Hazen

- University of Washington, David Stahl

- Montana State University, Matthew Fields

Functional Genomics and Imaging Core:

- LBNL, Jay Keasling and Aindrila Mukhopadhyay

- University of Missouri-Columbia, Judy Wall

- Southern Illinois University, Kelly Bender

- Sandia National Laboratory, Anup Singh

- $\quad$ Oak Ridge National Laboratory, Martin Keller

- University of Oklahoma, Jizhong (Joe) Zhou

Computational and Systems Biology Core:

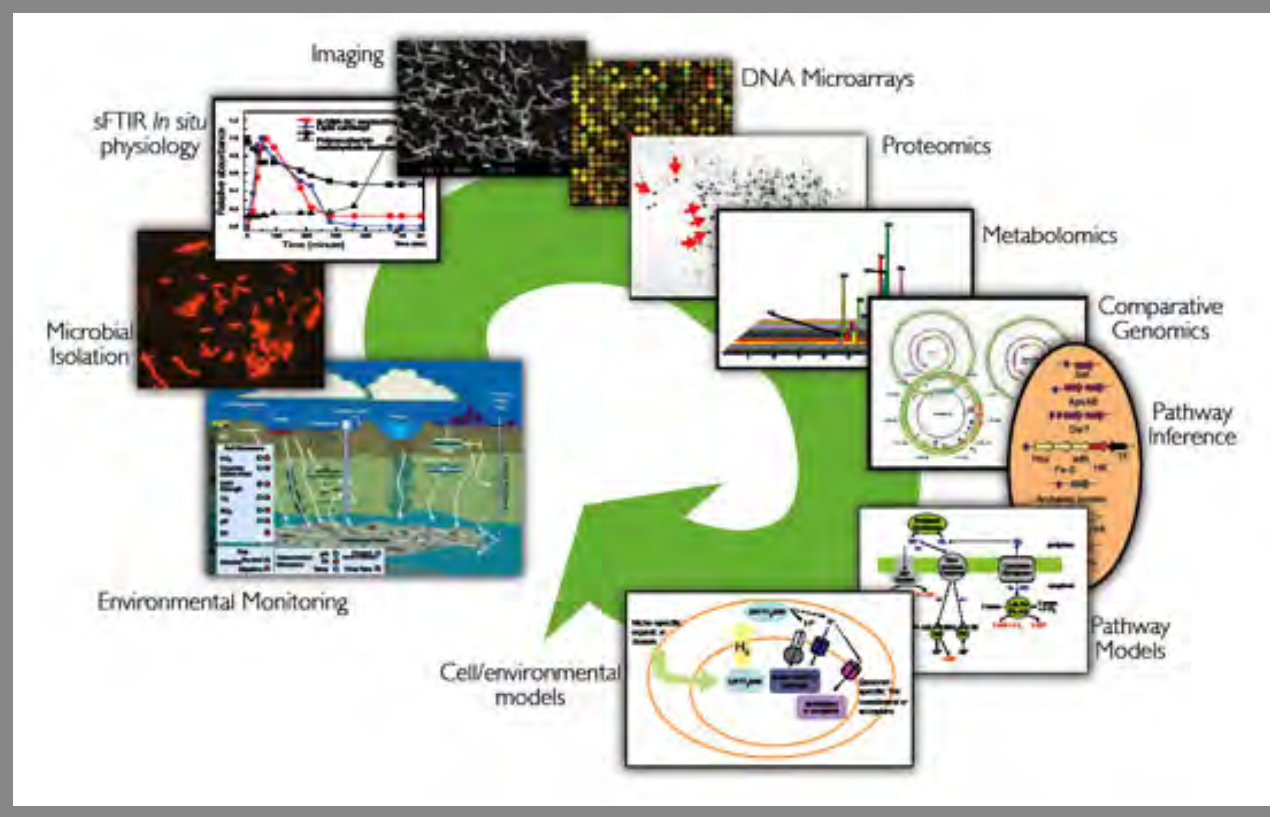

- $\quad$ LBNL: Adam P. Arkin, Inna Dubchak, Paramvir Dehal

- MIT: Eric Alm 